\title{
Thermoelectric Effects in Carbon Nanotube Quantum Dot in the Kondo Regime
}

\author{
D. KRYCHOWSKI* AND S. LIPIŃSKI \\ Institute of Molecular Physics, Polish Academy of Sciences \\ Smoluchowskiego 17, 60-178 Poznań
}

\begin{abstract}
Thermoelectric effects in a carbon nanotube quantum dot in the Kondo regime are studied by the equation of motion method. The thermopower is highly sensitive to the structure of the spectral density near the Fermi edge. For temperatures close to the Kondo temperature the thermopower approaches a local minimum, which becomes sharper for orbital energies closer to the Fermi level. For higher temperatures the tjermopower increases, but in the temperature range corresponding to charging energy it again drops and achieves significant negative values. We examine the consequences of symmetry breaking of spin-orbital SU(4) system on thermopower.
\end{abstract}

PACS numbers: 73.63.KV, 72.10.Fk, 73.63.-b, 65.80.+n

\section{Introduction}

A recent observation of the spin Kondo contribution to the thermopower (TEP) in lateral semiconducting quantum dot (QD) [1] has motivated theoretical investigations in this field (e.g. [2,3]). Among earlier papers on this subject most comprehensive is the paper of Bose and Fazio [4]. For weak coupling to the leads finite-length carbon nanotube behaves like a quantum dot (CNTQD) [5]. Despite the increasing interest in the thermoelectric characteristics of carbon nanotubes $[6,7]$, which is stimulated by potential applications of these systems as thermoelectric converters, the measurements of TEP of CNTQDs in the Kondo regime have not yet been reported. Experimentally, the main problem in performing TEP measurements on nanostuctrures is how to obtain a sizeable temperature difference across a small device.

In this paper we are interested in the study of thermoelectric effects induced by spin and orbital correlations of CNTQDs in the Kondo regime. For vanishing

*corresponding author; e-mail: krychowski@ifmpan.poznan.pl 
magnetic field and orbitally degenerate states the Kondo effect in CNTQDs appears simultaneously in spin and orbital sectors resulting in SU(4) Fermi liquid ground state with totally entangled spin and orbital degrees of freedom [8]. The Kondo resonance for this symmetry is much broader than for a conventional SU(2) system of the same position of the dot energy and its centre is shifted from the Fermi level towards higher energies. The symmetry breaking perturbation modifies the Kondo peak, in the weak case it induces a dip in the resonance peak and for strong perturbation the peak splits into two peaks. Whereas the linear conductance probes only the density of states (DOS) at the Fermi level, the linear TEP supports complementary information. TEP depends sensitively on the energy dependence of DOS in the range of temperature broadening. Symmetry breaking can be caused by different reasons. We examine the influence of magnetic field and polarization of electrodes.

\section{Model}

In the present considerations we discuss the Kondo physics of small semiconducting CNTQDs. For short nanotubes with well-separated energy levels it is enough to restrict at low temperatures to the single shell. The dot is modeled by double orbital Andreson Hamiltonian with intraorbital $U$ and interorbital Coulomb interaction parameter $U_{1}$ :

$$
\begin{aligned}
& H=\sum_{k \alpha m \sigma} E_{k \alpha m \sigma} c_{k \alpha m \sigma}^{+} c_{k \alpha m \sigma}+\sum_{k \alpha m \sigma} t_{k \alpha m \sigma}\left(c_{k \alpha m \sigma}^{+} d_{m \sigma}+\text { c.c. }\right) \\
& +\sum_{m \sigma} E_{m \sigma} d_{m \sigma}^{+} d_{m \sigma}+\sum_{m} U n_{m \uparrow} n_{m \downarrow}+\sum_{\sigma \sigma^{\prime}} U_{1} n_{1 \sigma} n_{-1 \sigma^{\prime}} .
\end{aligned}
$$

The energies of orbital states $(m= \pm 1)$, corresponding to clockwise and counterclockwise wrapping modes of CNTQD [9], are given by $E_{m \sigma}=E+\mu_{\mathrm{orb}} m h \cos (\theta)+$ $\sigma h+\sigma h_{\mathrm{SDIPS}}$, where $\theta$ specifies the orientations of magnetic field $h$ relative to the nanotube axis, $\mu_{\text {orb }}$ is the orbital moment, and $h_{\text {SDIPS }}$ denotes the effective, polarization dependent magnetic field describing spin dependence of interfacial phase shifts (SDIPSs) [9] (we set $g=\mu_{\mathrm{B}}=k_{\mathrm{B}}=1$ ).

The linear TEP deduced from nonequilibrium Keldysh technique with the use of Ng's ansatz $[10,11]$ is given by

$$
S=-\frac{1}{e T} \frac{L_{12}}{L_{11}}
$$

where the linear response coefficients are

$$
\begin{aligned}
& L_{11}=\frac{2 T}{h} \sum_{m \sigma} \int_{-\infty}^{+\infty} \mathrm{d} \omega\left(\frac{\partial f_{\alpha}(\omega)}{\partial \mu}\right)_{T} \Gamma_{\sigma} \operatorname{Im}\left[G_{m \sigma}^{+}(\omega)\right], \\
& L_{12}=\frac{2 T^{2}}{h} \sum_{m \sigma} \int_{-\infty}^{+\infty} \mathrm{d} \omega\left(\frac{\partial f_{\alpha}(\omega)}{\partial T}\right)_{\mu} \Gamma_{\sigma} \operatorname{Im}\left[G_{m \sigma}^{+}(\omega)\right] .
\end{aligned}
$$

$G_{m \sigma}^{+}$denotes the retarded Green function for the orbital $m, f_{\alpha}$ are the Fermi dis- 
tribution functions of electrodes, and $\Gamma_{\sigma}=\left(\Gamma_{L \sigma} \Gamma_{R \sigma}\right) /\left(\Gamma_{L \sigma}+\Gamma_{R \sigma}\right)$ is the effective coupling parameter. $\Gamma=\Gamma_{\uparrow}+\Gamma_{\downarrow}$ is taken as the energy unit. The Green functions are found by the equation of motion method using the self-consistent decoupling procedure proposed by Lacroix [12].

\section{Numerical results and discussion}

We present numerical results for $U=10$ and $U_{1}=8$ and the orbital moment $\mu_{\mathrm{orb}}=10$. Figure 1 presents TEP as a function of temperature for three different level positions and hence different Kondo temperatures. For $T \sim T_{\mathrm{K}}$ TEP approaches a local minimum and a monotonic shift of its position is observed with bringing the dot energy closer to the Fermi level. The depth of the minimum decreases with the decrease in $T_{\mathrm{K}}$ and for elevated temperatures TEP increases and eventually changes the sign.

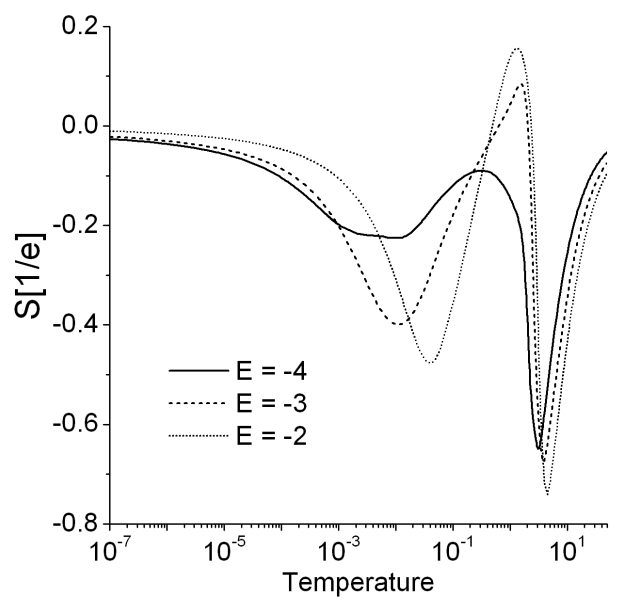

Fig. 1. Linear TEP $S(T)$ for different level positions.

This signals the disappearance of the Kondo correlations. For higher temperatures another pronounced minimum of TEP is observed, which is mainly due to the Coulomb resonances $\left(E+U, E+U_{1}\right)$. In the following we restrict ourselves to the Kondo regime. Figure 2 illustrates the filled anisotropy of temperature dependence of thermopower. Field perpendicular to the tube axis $h_{\mathrm{P}}$ breaks only the spin degeneracy, whereas axial field $h_{\mathrm{A}}$ breaks both spin and orbital degeneracy.

For perpendicular orientation the low temperature TEP Kondo minimum is hardly resolvable and the second minimum occurring at higher temperatures corresponds to satellite peaks in DOS resulting from inter- and intraorbital spin flipping transitions. The field induced changes of DOS (inset of Fig. 2a) reflect in the difference of the spin resolved contributions to TEP. For spin up a clear minimum of $S(T)$ is observed for $T \approx 2 \mathrm{~h}$, whereas for spin down only inflection 

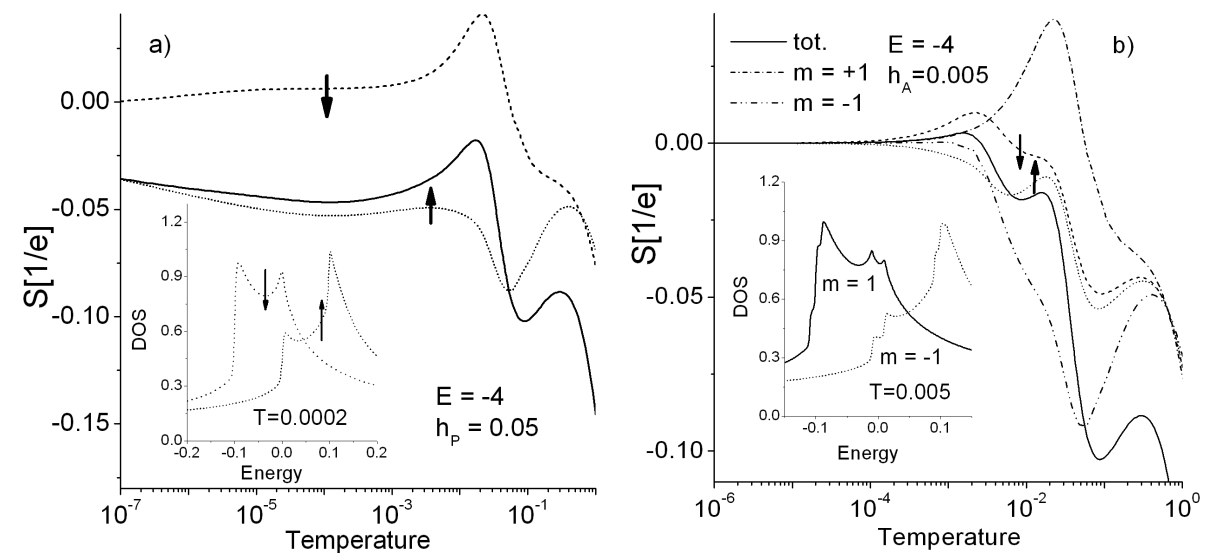

Fig. 2. TEP of CNTQD placed in a magnetic field (a) perpendicular field case: total TEP and spin resolved contributions, inset shows spin dependent DOS; (b) parallel field case: $S(T)$ together with orbital and spin contributions, inset shows the partial orbital DOS.
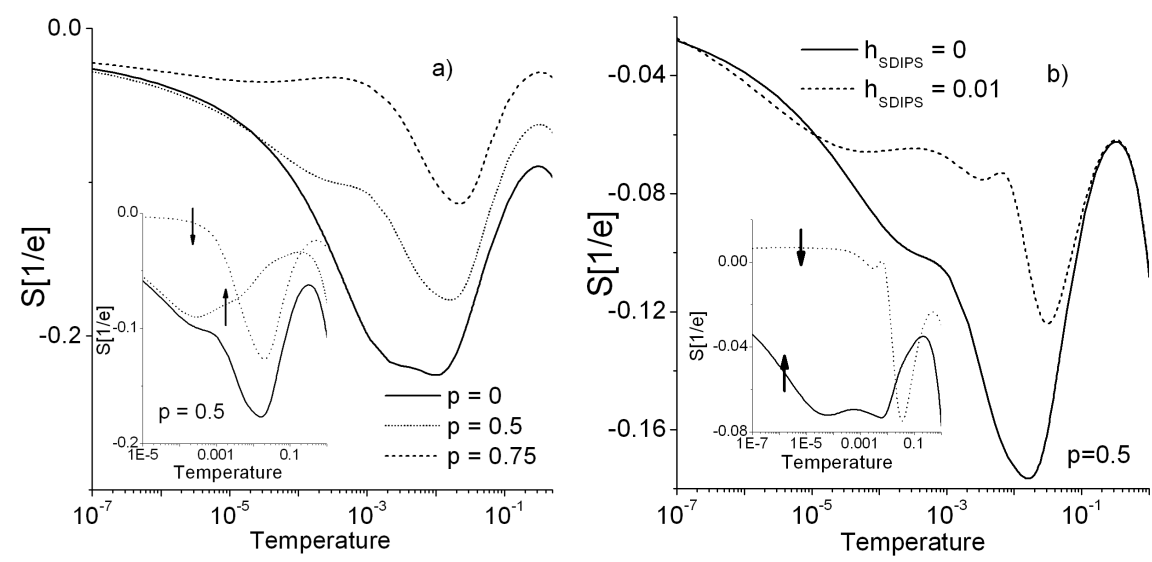

Fig. 3. TEP of CNTQD with polarized electrodes (a) $S(T)$ for different values of polarization, inset shows the spin decomposition of $S(T)$; (b) $S(T)$ for the dot with polarized electrodes calculated with spin-dependent dot levels, inset shows the spin contributions to $S(T)$.

is seen in this region. For axial field (Fig. 2b) the main minimum occurs for temperatures of an order of magnitude higher $(T \approx 20 \mathrm{~h})$, which is a consequence of large value of orbital magnetic moment $\left(\mu_{\mathrm{orb}} \approx 10 \mu_{\mathrm{B}}\right)$. This minimum corresponds to the orbital pseudospin fluctuations (inset of Fig. 2b). Another minimum of $S(T)$ is also seen for lower temperatures $(T \approx 2 \mathrm{~h})$ and it results from spin fluctuations.

Figure 3 presents an influence of another symmetry breaking perturbation - the spin polarization of electrodes. For a large enough polarization, defined as the relative difference of tunneling rates for spin up and down electrons 
$\left(p=\left(\Gamma_{\uparrow}-\Gamma_{\downarrow}\right) /\left(\Gamma_{\uparrow}+\Gamma_{\downarrow}\right)\right)$, the Kondo peak splits, which introduces the minima in the spin resolved contributions to TEP. The minima are located at different temperatures for each spin orientation (Fig. 3a). The interface between a ferromagnet and carbon nanotube can differently scatter electrons with spin parallel or antiparallel to the magnetization of ferromagnet inducing an effective spin splitting $\left(\sigma h_{\text {SIDPS }}\right)$. This splitting can lead to an additional low temperature structure as shown in Fig. 3b.

Summarizing, the present paper shows that studying the low temperature thermoelectric power allows one to probe the slope of the Kondo resonance and its reconstruction under the symmetry breaking perturbations and thus it is a complementary method to differential conductance to study the low energy DOS.

\section{Acknowledgments}

The work has been supported by the EU grant CARDEQ under contract IST-021285-2.

\section{References}

[1] R. Scheibner, H. Bahmann, D. Reuter, M.N. Kiselev, L.W. Molenkamp, Phys. Rev. Lett. 95, 176602 (2005).

[2] M. Krawiec, K.I. Wysokiński, Phys. Rev. B 73, 075306 (2006).

[3] R. Sakano, T. Kita, N. Kawakami, cond-mat/0701533 (to be published in Physica E).

[4] D. Bose, R. Fazio, Europhys. Lett. 56, 576 (2001).

[5] S. Sapmaz, P. Jarillo-Herrero, L.P. Kouvenhoven, H.S.J. Van Der Zant, Semicond. Sci. Technol. 21, 52 (2006).

[6] M.C. Llaguno, J.E. Fisher, A.T. Johnson, J. Hone, Nano Lett. 4, 45 (2004).

[7] J.P. Small, K.M. Perez, P. Kim, Phys. Rev. Lett. 91, 256801 (2003).

[8] M. Choi, R. Lopez, R. Aquado, Phys. Rev. Lett. 95, 0672041 (2005).

[9] A. Cottet, T. Kontos, S. Sahoo, H.T. Man, M.S. Choi, W. Belzig, C. Bruder, A.F. Morpurgo, C. Schönenberger, Semicond. Sci. Technol. 21, S78 (2006).

[10] T.K. Ng, Phys. Rev. Lett. 76, 487 (1996).

[11] Bing Dong, X.L. Lei, J. Phys., Condens. Matter 14, 11747 (2002).

[12] C. Lacroix, J. Phys. F, Metal Phys. 11, 2389 (1998). 